\title{
Las fuentes orales en la Educación Secundaria: motivación y aprendizaje de la Historia*
}

\section{Oral Sources in Secondary Education: Motivation and History Learning}

\section{Sara Prades Plaza}

Universitat Jaume I

Resumen: La experiencia que aquí se expone demuestra que el uso de fuentes orales fomenta el interés del alumnado de Educación Secundaria por la Historia y afianza sus conocimientos. Mediante entrevistas a sus allegados el alumnado se ha acercado a los protagonistas del pasado, evitando una visión despersonalizada de los tiempos pretéritos que dificulte la comprensión de la complejidad de la historia. Además, el tratamiento de estas fuentes históricas ha contribuido a una educación más próxima a la cultura científica, al desarrollar habilidades reflexivas y de investigación entre los alumnos. La comparación de los resultados de aprendizaje de estos alumnos con otros que no habían tratado las fuentes orales ha confirmado nuestras hipótesis.

Palabras clave: Didáctica de la Historia, ESO, metodología didáctica, fuentes orales, motivación, franquismo.

Abstract: The experience presented here demonstrates that the use of oral sources promotes the interest of students in secondary education by history and strengthens their knowledge. Through interviews with those close to students has approached the protagonists of the past, avoiding a depersonalized vision of the past times that hinders understanding of the complexity of the story. Furthermore, treatment of these historical sources has contributed to closer to scientific culture education, research and develop reflective skills among students. The comparison of the learning outcomes of these students with others who had untreated oral sources have confirmed our hypothesis.

Keywords: Teaching History, ESO, teaching methodology, oral sources, motivation, Francoism.

(Fecha de recepción: diciembre, 2014, y de aceptación: febrero, 2016)

DOI: 10.7203/DCES.30.4478

* La autora participa en el proyecto de investigación "Derechas y nación en la España contemporánea. Culturas e identidades en conflicto" (HAR2014-53042-P) financiado por el Ministerio de Economía y Competitividad. Quisiera agradecer las valiosas aportaciones de David Parra Monserrat a este trabajo. 


\section{Por qué introducir \\ las fuentes orales en la ESO}

Después de varios años de docencia en Educación Secundaria Obligatoria, constatamos la percepción de un gran número de estudiantes de la materia de Historia como una enseñanza aburrida y alejada de sus intereses; considerada por ellos, en definitiva, inútil. Algunos incluso consideraban que la Historia era una materia alejada del método científico, puesto que entendían que para aprenderla era suficiente con retener muchos datos que, a su parecer, tenían poco significado en la actualidad. Entre otras razones esgrimidas por los alumnos, su desmotivación por aprender esta asignatura venía dada por la incapacidad de los docentes de conectarla con la realidad que el alumnado vivía.

Seguramente esto era consecuencia de que en la mayoría de casos, como pudimos comprobar, aún se daba el aprendizaje tradicional transmisivo. Esta metodología didáctica, basada en el trabajo individual del alumno, la impartición de la clase magistral por parte del docente y la no intervención del discente a menos que se lo requiera el profesor dificulta, entre otras cuestiones, la adquisición de la competencia social y ciudadana por parte de los alumnos, al no fomentar en ellos la capacidad de diálogo, creatividad, reflexión y participación (Aubert et al., 2008).

Pero sus inconvenientes no afectan solo al alumno, sino que esta práctica crea en el profesor una sensación de carga y desmotivación, por considerarle el único responsable del aprendizaje del alumno. Ambas razones, el desinterés del alumnado y la preeminencia de la instrucción transmisiva, nos llevaron a reflexionar sobre la urgencia de un cambio en el proceso de enseñanza-aprendizaje de la Historia, que nos condujo al interés por introducir, entre otros recursos, las fuentes orales en la Educación Secundaria Obligatoria. Entendemos que la introducción y manejo de estas fuentes históricas contribuyen a facilitar la comprensión de los procesos sucedidos en contextos lejanos en el tiempo, puesto que los métodos en que el alumno es un sujeto activo posibilitan una mayor consecución de objetivos de aprendizaje.

Ha sido ampliamente constatado que el uso de las fuentes históricas permite una aproximación más significativa al pasado, como demostró ya en los años ochenta el grupo "Historia 13-16" y su método por descubrimiento (Sallés, 2010). Igualmente, las fuentes orales desarrollan la capacidad de investigar en nuestros alumnos, fundamental en la disciplina histórica y poco trabajada, en general, en la Educación Secundaria Obligatoria (Prats, 2001, p. 26). De igual modo, gracias al tratamiento de las fuentes históricas, el alumnado se familiariza con la labor de construcción del conocimiento histórico mediante el método científico, a través del desarrollo de competencias como la interpretación histórica y el pensamiento crítico. En concreto, mediante las fuentes ora- 
les se contribuye a la comprensión de la Historia como una ciencia en construcción, abierta e inacabada, y conectada con sus protagonistas, las personas que han vivido el pasado que aprenden nuestros alumnos (Monteagudo, 2013).

Esta concepción de la Historia ha dado lugar en otros tiempos a encendidas controversias que han puesto en duda el estatus científico de esta disciplina. Actualmente, esto ya no es motivo de discusión, ya que no es sólo la búsqueda de hechos verdaderos lo que constituye una ciencia, sino la elaboración de conceptos, leyes y teorías. En efecto, el objeto de la Historia es verificar las pruebas materiales que sirven de apoyo a afirmaciones históricas y explicar la causalidad y la significación temporal, excluyendo anacronismos en las interpretaciones del pasado. Este objetivo se podrá conseguir gracias a los restos que hayan quedado del pasado, que denominamos fuentes históricas, cuyo análisis servirá de base para la elaboración del discurso histórico tras su tratamiento según una cuidada metodología. Enseñar a los adolescentes su método de análisis y, por ende, desarrollar en ellos actitudes críticas contribuirá a que en la vida adulta puedan razonar de manera autónoma sobre estas fuentes de conocimiento del mundo en que viven.

Además, con su tratamiento se adquiere el desarrollo de las Competencias Básicas, definidas como capacidades que permiten "poder lograr su realización personal, ejercer la ciudadanía activa, incorporarse a la vida adulta de manera satisfactoria y ser capaz de desarrollar un aprendizaje permanente a lo largo de toda la vida" (MEC, 2007, p. 678). En concreto, y sin dejar de lado las demás, gracias al tratamiento de las fuentes orales en el aula de Historia principalmente se desarrollan las competencias social y ciudadana, aprender a aprender, competencia cultural y artística y la autonomía e iniciativa personal.

De igual modo, mediante el uso de las fuentes orales se crea un espacio de intercambio generacional que permite la participación ciudadana en la escuela, el acercamiento de los alumnos a la sociedad en que viven y el desarrollo de actitudes empáticas por parte del alumnado hacia los mayores. Así, practican la tolerancia al comprender las opiniones de los entrevistados (Sepúlveda, 2000) y considerar a los ancianos como fuentes de saber y sujetos útiles para la sociedad a quien merece la pena escuchar, al tiempo que ahondan en su conocimiento personal. Adicionalmente, en las personas mayores entrevistadas la participación en este tipo de experiencias produce una sensación de enorme satisfacción al sentirse útiles como agentes implicados en el cambio de la sociedad, por contribuir a que los adolescentes de su entorno afiancen sus conocimientos históricos.

\section{Las fuentes orales: \\ lo viejo y lo nuevo}

El uso de las fuentes orales se ha dado sistemáticamente para el conocimiento 
histórico desde la década de 1960, cuando el sociólogo Paul Thompson inició la reivindicación de esta fuente histórica al entrevistar a varios individuos para conocer aspectos sobre su vida laboral y familiar. Pretendía encontrar datos acerca de cuestiones difícilmente reflejadas en los archivos como sus ideologías, sus comportamientos sociales y algunas prácticas de sociabilidad como las costumbres, etc. La información sobre estos aspectos la halló gracias a las fuentes orales. De este modo, se recuperaba mediante los testimonios orales la relevancia del individuo anónimo, del hombre corriente, en la explicación del pasado. Además, se concedía credibilidad a una fuente histórica accesible para todo el mundo, cuya explotación no es sólo patrimonio de los historiadores, pues cualquiera se puede acercar a un protagonista del pasado reciente y preguntarle cómo respondió ante una determinada situación (Thompson, 1988).

Un tiempo después, la trascendencia que adquirieron los trabajos en Francia de Joutard (1986) y, para el caso español, los de Ronald Fraser (1979), acabaron de legitimar a la fuente oral por su gran interés para la construcción del discurso histórico. Sobre todo, cabe destacar la utilidad de esta fuente histórica para llevar a cabo estudios de historia local, tal y como hicieron en sus pioneros trabajos Mercè Vilanova (1985) para el caso de dos municipios gerundenses en tiempos de la II República y la Guerra Civil y el estudio de Joan Miralles (1985) sobre Montuïri.
No obstante, para su tratamiento como fuente histórica debemos tener en cuenta que el olvido a menudo es tan importante como la memoria, como los silencios, como las incertidumbres a la hora de construir el relato sobre el pasado. De hecho, la forma de recordar un acontecimiento es tan importante como el recuerdo en sí mismo, por posibilitarnos conocer la interpretación que hace el protagonista del mismo. Por eso, gracias a las fuentes orales se contribuye a negar el determinismo histórico, ya que analizamos cómo han actuado diferentes sujetos ante un mismo contexto, aunque éste les haya condicionado.

En tiempos pasados, la subjetividad de la memoria fue considerada un problema para ser usada en ámbitos académicos, sobre todo cuando se creía que la palabra escrita era más digna de credibilidad. Hoy nadie duda de que tanto la fuente escrita como la oral siempre se deben de contrastar con otras fuentes, dado que la parcialidad no es únicamente un inconveniente de la oral. De hecho, todas las fuentes históricas que han quedado del pasado no son una muestra accidental de lo que originariamente existió, sino que han sido escogidas para que perduraran en el tiempo.

Entre los problemas que presenta la fuente oral cabe destacar que solamente puede contribuir al conocimiento de la historia contemporánea, acercando a los protagonistas del pasado a la explicación histórica. Igualmente, hay que tener en cuenta su temporalidad limitada, dado que tenemos dificultades para encontrar testigos de hechos que 
se remontan demasiado en el tiempo. A veces, se han de hacer entrevistas con una precaria preparación previa del entrevistador por la urgencia vital que se tiene, dado que el pasado recuperable mediante la evidencia oral se reduce día a día debido a la muerte de sus protagonistas. En otras ocasiones, las entrevistas no se pueden contrastar con otras fuentes orales porque ya no hay más testigos que nos puedan informar del hecho particular que estudiamos.

A pesar de los problemas de que adolece esta fuente histórica, son mayores las virtudes que presenta, razón por la cual en la década de los noventa fueron numerosos los intentos de introducir las fuentes orales en las enseñanzas regladas, dando lugar a distintas experiencias (Janer y Santos, 1990; Núñez, 1990; Sánchez, 1991). Se comprobó que este tipo de fuentes históricas solían despertar un enorme interés entre los alumnos de Historia, puesto que entraban en relación con protagonistas vivos del pasado que estudiaban (Benadiba y Plotinsky, 2009).

Por lo tanto, las fuentes orales contribuyen a que los estudiantes consideren que la Historia no es solo una asignatura obligatoria del sistema educativo, sino una materia totalmente necesaria para entender por qué las cosas son como son en el presente. Además, gracias a las fuentes orales los alumnos de las enseñanzas regladas se acercan al conocimiento de la historia cotidiana, la del hombre del día a día, alejado de los grandes personajes, acontecimientos y élites de poder. Mediante estas fuen- tes conocen el pasado que vivieron sus familiares, vecinos y conocidos, así como algunos procesos pretéritos poco reflejados en otros recursos didácticos por estar alejados de las esferas de poder. Pero para ello es importante no perderse en cuestiones triviales o anecdóticas y analizar cambios profundos sociales, económicos, ideológicos o políticos que afectaron al conjunto de la sociedad en que viven nuestros alumnos.

\section{Fuentes orales en cuarto de Educación Secundaria Obligatoria: la reconstrucción de la memoria del franquismo}

Nuestro principal objetivo era conocer si el uso de las fuentes orales en el aula de Historia contribuía a la motivación del alumnado y a la adquisición de competencias y conocimientos históricos. Con esto, buscábamos difundir el valor de las fuentes orales como recurso pedagógico para ser usado en el aula $y$, de esta forma, demostrar la mejora del aprendizaje de un periodo histórico mediante el cambio metodológico del proceso de enseñanza-aprendizaje.

En la experiencia participaron cincuenta y cinco alumnos de entre quince y diecisiete años de un centro público de la provincia de Castellón al que asisten alumnos de cinco municipios de la comarca de la Plana Baixa. Pertenecían a dos grupos de alumnos de cuarto de ESO, nivel educativo elegido por ser el único de esta etapa educativa en que se tratan contenidos de Historia Contem- 
poránea, susceptibles de ser abordados con fuentes orales.

Para conseguir los objetivos antes expuestos se diseñaron dos unidades didácticas con metodologías diferentes, con la intención de comparar los resultados obtenidos con cada una de ellas en que se trataría el mismo tema: el franquismo. Nuestra práctica se llevó a cabo siguiendo un método empíricoanalítico, usado en muchas investigaciones de ciencias sociales, para el que elaboramos los materiales que nos permitieron obtener la información con la que posteriormente extraer conclusiones. Para la recogida de datos los instrumentos de análisis empleados fueron la observación, los cuestionarios de conocimiento de ideas previas, diseñados de acuerdo con los objetivos de aprendizaje de la etapa educativa a que iban dirigidos, las entrevistas y una actividad final de síntesis que realizaron nuestros alumnos.

El cuestionario inicial (Cuadro I), que se pasó individualmente al alumnado, preguntaba sus datos personales y trataba de averiguar cuestiones sobre metodología en la clase de Historia y sus conocimientos previos sobre el franquismo. Este fue pasado a principios de año, cuando ya llevaban un trimestre cursando la asignatura de Historia. El cuestionario reveló que cada uno de los grupos de cuarto de ESO era heterogéneo por lo que respecta al género y localidad de procedencia, pero era homogéneo en cuanto a la edad y nivel de estudios. Además, puesto que ya conocíamos a los alumnos de ambos grupos del pri- mer trimestre, y a algunos de ellos de otros cursos escolares, sabíamos que los ritmos de trabajo de los dos grupos eran muy similares.

$\mathrm{Al}$ preguntar sobre si les gustaba o no la materia de Historia, en el grupo A contestaron afirmativamente el $68 \%$ del alumnado, mientras que en el grupo B solo al $40 \%$ les gustaba esta asignatura. De entre quienes decían apreciar esta materia, la mayoría contestaron que les gustaba porqué les interesaba el pasado, mientras que a quienes les disgustaba era porqué les resultaba aburrida. Seguidamente, preguntamos acerca de cómo se les había impartido la Historia hasta ese momento en la ESO. La respuesta mayoritaria que dio el $90 \%$ de los alumnos fue que el docente casi siempre seguía el libro de texto, aunque invitaba a participar a los alumnos en la clase, a pesar de que raras veces habían trabajado en grupo. No obstante, el $72 \%$ del alumnado contestó que el profesor a menudo usaba otros materiales distintos al manual escolar, como mapas, presentaciones Power Point, atlas, novelas, vídeos u otros libros.

Sobre conocimientos previos acerca del franquismo, el cuestionario mostró que la totalidad del alumnado tenía nociones, aunque algunas puntuales y anecdóticas, acerca de este periodo histórico. Gracias al cuestionario pudimos comprobar que ambos grupos de cuarto de ESO partían de ideas previas muy similares. Además, durante el primer trimestre se habían estado analizando las distintas fuentes históricas en 


\section{Cuadro I \\ Cuestionario Inicial}

(Fuente: Elaboración propia)

Nombre:

Grupo:

Edad:

Género:

Localidad en que vives:

\section{Contesta sinceramente a estas preguntas:}

1. ¿Te gusta la Historia?

2. ¿Por qué te gusta o por qué no?

3. ¿Te ha ido bien esta asignatura en cursos anteriores?

4. ¿Cómo han dado las clases de Historia tus profesores de la ESO?

5. ¿Te han animado a participar en la clase?

6. ¿Habéis trabajado en grupos o individualmente?

7. De los siguientes recursos, ¿cuáles usabais?: libro de texto, mapas, presentaciones Power Point, atlas, novelas, vídeos, otros libros distintos del manual.

\section{Explica con claridad qué sabes de las siguientes cuestiones:}

1. ¿Quién fue Francisco Franco?

2. ¿Qué es una dictadura?

3. ¿En el franquismo había libertad política y social?

4. ¿Qué es la autarquía?

5. ¿En casa, has oído hablar de cómo vivían tus abuelos cuando eran jóvenes?

6. ¿A qué se dedicaban tus abuelos? ¿Sabes cuáles eran sus condiciones laborales?

7. ¿Cómo era su escuela? ¿Qué asignaturas estudiaban? ¿Cómo eran las relaciones con sus profesores?

8. ¿Qué hacían para divertirse?

9. ¿Cómo comían?

10. ¿Cómo se podían relacionar las personas de diferente sexo antes del matrimonio? 
ambos grupos de alumnos, por lo que todos estaban familiarizados con su uso, sus posibilidades, sus virtudes e inconvenientes de su tratamiento.

Conocidos estos datos, comenzamos a trabajar la unidad didáctica del franquismo, introduciendo el uso de fuentes orales en el grupo B, pero no en el A, con el objetivo de comparar los resultados al final de la experiencia. Se decidió introducir en este grupo este tipo de fuentes porque eran quienes inicialmente partían de un mayor desinterés por la materia de Historia. En el grupo A, el profesor seguía siendo el guía del proceso de enseñanza-aprendizaje, conduciendo a los alumnos a un aprendizaje autónomo basado en fuentes escritas y en el uso del libro de texto, pero no en fuentes orales.

Seguidamente, planteamos a nuestros alumnos del grupo B la experiencia e hicimos un sondeo entre ellos para encontrar al tipo de personas que nos podía resultar interesante entrevistar, buscando a los más adecuados para nuestro estudio de entre sus allegados, ya fuesen familiares, conocidos, vecinos o amistades. Como en toda experiencia con fuentes orales, consideramos interesante encontrar perspectivas enfrentadas para el análisis de algunas cuestiones, por ejemplo de mujeres y hombres, pertenecientes a diferentes clases sociales de una misma localidad, de diversas edades, de diferentes opiniones políticas o pertenecientes a grupos de vencedores y vencidos en la Guerra Civil. Además, intentamos averiguar si entre los allegados de nuestros alum- nos había gente de especial interés por tener una memoria privilegiada o porque sus experiencias habían sido críticas o excepcionales durante la etapa estudiada. Otros entrevistados fueron escogidos como representativos de un grupo más amplio.

Una vez elegidos los individuos a entrevistar, intentamos averiguar todo lo que pudimos sobre ellos, ya que era sumamente importante hacerse una idea clara de lo que nos iban a contar. Los entrevistados fueron dos labradores de setenta y dos y setenta y cinco años respectivamente, un panadero de ochenta años, una costurera de setenta y seis, un sargento de la guardia civil de setenta y nueve, una ama de casa de setenta y ocho, un maestro de escuela de setenta, un enfermero que había sido alcalde durante el franquismo de ochenta y tres años, el dueño de un taller de reparación de coches de setenta y siete años y una profesora de Educación Física de sesenta y cuatro años que había pertenecido y trabajado para la Sección Femenina de Falange Española. En el momento de realizar las entrevistas, todos estaban jubilados.

Asimismo, procuramos que nuestro alumnado conociese previamente el contexto histórico en el que vivieron las personas a entrevistar, para discernir los hechos acontecidos de la opinión de los encuestados. En este sentido, tratamos de antemano en clase las distintas etapas políticas y económicas por las que pasó el régimen de Franco, cómo era la vida cotidiana durante las distintas décadas del franquismo y las relaciones 
entre géneros, entre otras cuestiones. Gracias a este conocimiento previo de algunas de las cuestiones que íbamos a tratar, las fuentes orales sirvieron para contrastar o complementar hipótesis sobre los procesos estudiados en clase.

También resultó fundamental que los entrevistadores, es decir, nuestros alumnos, fuesen capaces de presentarse como interlocutores válidos, identificándose con los espacios físicos y de relaciones sociales vivenciados por el narrador, ya que se ha comprobado que las fuentes orales se dirigen preferentemente a quienes se ubican en relación a su espacio, facilitando enormemente la recuperación de su memoria. En todo caso, advertimos a nuestros alumnos que se mostrasen respetuosos e interesados por los entrevistados, debiendo ser comprensivos con las confesiones más íntimas. De este modo, en grupos de dos o tres alumnos visitaron a los individuos a entrevistar, con quienes compartían relaciones de vecindad, amistad o parentesco.

Aunque se puedan poner en práctica diferentes modelos de entrevista, desde la aproximación más amistosa e informal hasta el cuestionario cerrado y riguroso, se debe de aplicar la variante que en cada momento se adapte mejor a la personalidad del entrevistado y a la finalidad de la entrevista. En cualquier caso, el acceso a las fuentes orales requiere de una cuidadosa técnica, en que cabe tener en cuenta la actitud y aspecto del entrevistador, el lugar de la entrevista y la duración de la misma, el planteamiento de preguntas breves con palabras sencillas y evitar sugerir respuestas o plantear preguntas capciosas.

Conocidos estos aspectos por parte de nuestros alumnos, llevaron cuestionarios estructurados en que se proponían una serie de preguntas que consensuamos en clase (Cuadro II). No consideramos obligatorio ceñirse a las mismas en caso de que el entrevistado hablase fluidamente, pero podían servir de ayuda ante individuos más reservados o con peor memoria. Las entrevistas, realizadas fuera del horario escolar, siempre comenzaban preguntando la edad y profesión para posteriormente averiguar sus recuerdos sobre la dictadura de Franco.

La mayoría de entrevistas fueron grabadas en vídeo, para valorar cuestiones de lenguaje corporal que no aparecen en frases ni en palabras como pueda ser el tono de la voz, los cambios posturales ante preguntas incómodas, los silencios o la dificultad para recordar determinados episodios. Alguna grabación se realizó con una asunción implícita de confidencialidad, cuestión que fue respetada por nuestros alumnos, de igual forma que, en estos casos, las citas se hicieron con carácter anónimo. En la mayor parte de ocasiones, se contó explícitamente con la autorización de los entrevistados para usar sus palabras en nuestro provecho.

Una vez tuvimos registradas las entrevistas, se transcribió el material en su totalidad, sin descuidar los sentimientos expresados ni los silencios. Con las entrevistas transcritas, nues- 


\section{Cuadro II \\ Cuestionario a responder por parte de los entrevistados}

(Fuente: Elaboración propia)

Nombre:

Fecha y lugar de nacimiento:

Profesión:

Estudios:

Estado Civil:

Creencias religiosas:

Afiliación política, sindical o asociativa:

1. ¿Recuerda usted la posguerra?

2. ¿Cómo eran las relaciones en su familia? ¿Cuántos vivían en su casa? ¿Qué mobiliario tenían?

3. ¿Se hablaba en casa de política?

4. ¿Pasó usted hambre en los años cuarenta o cincuenta?

5. ¿Qué diversiones tenían en la posguerra? ¿Y durante los años cincuenta y sesenta?

6. ¿Conoce a alguien que fuese detenido por las fuerzas de seguridad? Si fue así, ¿de qué se le acusaba? ¿Provocaban rechazo, miedo, lástima o indiferencia?

7. ¿Qué recuerda con mayor claridad del franquismo?

8. ¿Opina que fue una buena época para su pueblo? ¿Qué recuerda de aquella época?

9. ¿Cómo era su trabajo: horas, salario y condiciones? ¿Cómo lo obtuvo?

10. ¿Cuándo percibió usted que había habido un cambio económico? ¿Recuerda cuándo aparecieron los electrodomésticos, alcantarillado, coches, se dio un acceso generalizado a los estudios universitarios o un aumento del turismo?

11. Cuando mejoró la situación económica, ¿se produjeron cambios en su trabajo, en cuanto a salario, horario o condiciones?

12. ¿Cómo eran las relaciones con el sexo contrario antes del matrimonio? ¿Y después? ¿Cambió esto a lo largo del franquismo?

13. ¿Cómo eran las celebraciones familiares o locales? 
tros alumnos intentaron encontrar ideas comunes al colectivo social al que pertenecía el informante, autorrepresentaciones, búsquedas de coherencia de vida desde el presente del entrevistado, olvidos para huir de la mala conciencia, etc. e intentaron traducir las limitaciones de la expresión verbal de cada sujeto. Muchas veces los entrevistados confundían datos y fue habitual que condensasen dos hechos similares en un solo recuerdo.

Posteriormente, los alumnos evaluaron el material recogido para averiguar si el informante tendía a la mitificación, al olvido o a la elisión. Con frecuencia, las fuentes orales mostraron discrepancias entre ellas, correspondiendo en ocasiones a varios puntos de vista que daban múltiples indicios sobre lo acontecido. En otras ocasiones, la información no se pudo contrastar, al no poder proceder de ninguna otra fuente, como en el caso del estudio de la relación de la vida doméstica de una familia determinada de la que sólo nos pudo informar quien lo había vivido. En cualquier caso, nuestros alumnos aprendieron a diferenciar la Historia de las memorias de vida de los entrevistados, discerniendo los pasajes subjetivos del conocimiento sobre el periodo que tenían.

\section{Resultados}

Al finalizar la unidad didáctica, cada alumno realizó una actividad de síntesis en que había de demostrar qué había aprendido sobre el franquismo. La metodología de aprendizaje había sido diferente en el grupo A y en el B, pero para evaluar la eficacia de la introducción de las fuentes orales en el aula de Secundaria era necesario que la actividad de recapitulación fuese la misma. Los resultados fueron considerablemente más satisfactorios en el grupo $\mathrm{B}$, que había trabajado con fuentes orales, que entre quienes no lo habían hecho, puesto que relacionaban el tema con aspectos que se habían estudiado anteriormente, mostraban empatía histórica y una mayor capacidad argumentativa.

En la actividad de síntesis (Cuadro III), al preguntar por la existencia de similitudes y diferencias del franquismo respecto de otras dictaduras antiliberales que se habían dado en la Europa de la Edad Contemporánea, en el grupo que no había trabajado las fuentes orales se relacionó el franquismo con el fascismo, el nazismo y la dictadura de Primo de Rivera. En el grupo que había analizado fuentes orales, además de hacer referencia a los anteriores regímenes antiliberales, hubo alumnos que lo relacionaron con el autoritarismo bonapartista, al establecer semejanzas en cuanto a la intromisión de los militares en la política. También en este grupo, una alumna relacionó el comportamiento de la Iglesia con el que se había dado en las monarquías absolutas, puesto que sirvió de elemento de legitimación del régimen de Franco.

En cuanto a la empatía histórica, los resultados del grupo que había trabajado con fuentes orales fueron más satisfactorios que los de aquellos que no lo 


\section{Cuadro III \\ Actividad de síntesis a responder por los alumnos al finalizar la unidad didáctica}

(Fuente: Elaboración propia)

Nombre:

Grupo:

Profesión:

Estudios:

1. Explica las similitudes y diferencias del franquismo con otras dictaduras antiliberales de la Europa contemporánea ( 2 puntos)

2. ¿Por qué Franco no fue derrotado? (2 puntos)

3. ¿Cuáles son las diferencias entre una democracia y una dictadura? (2 puntos)

4. Comenta el siguiente texto histórico (2 puntos)

"Queremos una España fraternal, una España laboriosa y trabajadora, donde los parásitos no encuentren acomodo. Una España sin cadenas ni tiranías judaicas, una nación sin marxismo ni comunismo destructores, un Estado para el pueblo, no un pueblo para el Estado. Una España sin bandos políticos en constante guerra, sin preponderancias parlamentarias ni asambleas irresponsables. Queremos una España grande, fuerte y unida, con autoridad, con dirección y orden".

Discurso de Francisco Franco Bahamonde, 1939

5. Define los siguientes conceptos con precisión (2 puntos): golpe de Estado, Falange Española Tradicionalista y de las Jons, Autarquía, Tecnócrata.

habían hecho, puesto que respondieron de modo distinto a la pregunta: “ $¿$ Por qué Franco no fue derrotado?" Los primeros, al haber conocido las esperanzas, intereses, desgracias y experiencias de todo tipo de quienes vivieron la dictadura, entendían sus respuestas y silencios ante el régimen de Franco. Los segundos, en la mayoría de casos, se mostraban muy críticos con los protagonistas del pasado por haber dejado que el dictador muriese en la cama después de casi cuarenta años de gobierno. 
Además, los alumnos del grupo que había trabajado las fuentes orales entendieron con mayor claridad las bondades de la democracia, al comparar las libertades de que hoy gozan con la situación cotidiana de los entrevistados durante la dictadura, que llegó a impresionar a nuestro alumnado por la enorme restricción de libertades que sufrían los jóvenes en los años cuarenta.

Por último, se pasó a ambos grupos de alumnos un segundo cuestionario en que se volvía a preguntar, entre otras cuestiones, si les resultaba interesante el estudio del pasado, con la finalidad de averiguar si su percepción de la Historia había cambiado después del uso de las fuentes orales. Gracias al cuestionario final y a la actividad de síntesis, que hicieron de forma idéntica ambos grupos de alumnos, se evaluó la eficacia de la introducción de las fuentes orales para afianzar conocimientos históricos y para generar interés entre nuestro alumnado por el estudio del pasado.

Puesto que nuestros principales objetivos eran motivar al alumnado y verificar la incidencia de los métodos en la enseñanza de la Historia, lo primero que analizamos fue la existencia o no de diferencias entre los resultados obtenidos en los dos métodos empleados. A pesar de las conclusiones extraídas, somos conscientes de que no es una muestra suficientemente amplia para dar lugar a generalizaciones.

Del cuestionario, es destacable que los alumnos del grupo B, donde antes de empezar a trabajar con fuentes ora- les había un $60 \%$ de alumnado desinteresado por nuestra materia, habían superado ahora en interés por la Historia a los del grupo A, puesto que del B había un 78\% que afirmaba gustarle la Historia. Como se ha dicho más arriba, el hecho que nos empujó a interesarnos por el uso de las fuentes orales en el aula de secundaria fue la detección de una elevada desmotivación entre el alumnado. La implicación de nuestros alumnos en esta iniciativa es una prueba del interés despertado por la misma así que, al menos como elemento motivador, la experiencia puede calificarse de exitosa. La satisfacción de nuestros alumnos al terminar la unidad didáctica y la oportunidad de haber hecho hablar a sus allegados sobre un pasado que, en ocasiones, había sido traumático fue una valiosa aportación a nuestra tarea docente.

Además, comprendieron que la Historia no era solamente una materia a estudiar, sino que formaba parte de sus vidas, dado que el pasado había condicionado las vivencias de sus familiares y vecinos. La Historia, que normalmente parecía a nuestros alumnos una asignatura impersonal y alejada de sus intereses, de repente les resultaba más familiar y cotidiana que nunca, por haber constituido el fundamento de la realidad presente de sus pueblos, de forma que se dio, en nuestra opinión, un aprendizaje verdaderamente significativo.

Gracias a esta experiencia pudimos comprobar cómo los alumnos que trabajaron con fuentes orales percibieron en 
mayor medida el sentido del aprendizaje de la Historia. A partir del diálogo con los protagonistas del pasado, multiplicaron las conexiones entre la materia de Historia y su realidad, logrando mayor nivel de comprensión no solo del pasado, sino también del presente en que vive el alumnado y el entrevistado. De igual modo, activaban la solidaridad y empatía con los actores del pasado.

Como tradicionalmente nuestros alumnos habían aprendido la Historia mediante la clase dirigida por el profesor, al introducirles el trabajo con fuentes orales algunos mostraron resistencias a romper con su rutina. El desconocimiento del método de trabajo de las fuentes orales implicó la inversión de algunas sesiones para aprender a tratarlas y convencerles de la conveniencia de esta metodología de trabajo, pues los métodos que tradicionalmente habían utilizado les resultaban más agradables y les parecían más eficaces.

Además, si consideramos que la difusión del conocimiento histórico en nuestra sociedad no solamente se da gracias a los aprendizajes adquiridos en las enseñanzas regladas, sino que en muchísimas ocasiones es originado por la visita a museos, exposiciones, por la información aparecida en medios de comunicación, ya sea prensa, radio, cine o televisión, por los comentarios de protagonistas del pasado, por la lectura de novelas históricas, etc., resulta de suma importancia el tratamiento de las ideas adquiridas por estos cauces. De este modo, nuestros estudiantes han aprendido a discernir entre juicios subjetivos de un novelista, cineasta, periodista o ciudadano y los acontecimientos del pasado, pero además han adelgazado la brecha existente entre el conocimiento histórico académico y el pasado que se dio en sus localidades.

Estas son las razones por las que consideramos de gran importancia la integración de las fuentes orales en las enseñanzas regladas, de forma que el alumnado sepa tratarlas y aprovecharlas adecuadamente. Así, este trabajo ha pretendido contribuir a evitar que el discurso histórico aparecido en la sociedad pueda parecer contradictorio, o incluso opuesto, al enseñado por el profesor de Historia. En nuestra opinión, esto fomenta el interés del alumnado por nuestra materia, al observar que la Historia es omnipresente en nuestra sociedad.

Mediante su introducción en las aulas de Educación Secundaria hemos pretendido que nuestros alumnos tomen consciencia del cambio historiográfico acaecido en las últimas décadas, consistente en no otorgar mayor credibilidad ni importancia a las fuentes documentales conservadas en archivos que, por lo general, son testimonio de la perspectiva de las élites masculinas que tradicionalmente han detentado el poder en las instituciones políticas, eclesiásticas o militares (Vilanova, 1994, p. 34). De este modo, el uso de estas fuentes históricas contribuye a erradicar el menosprecio de las propias experiencias vitales por parte de la mayoría de la población que ha vivido ajena a los cargos de poder institucional (Jardón, 
López y Soutelo, 1998). Gracias a su tratamiento, el alumnado puede acceder a los protagonistas reales del pasado, evitando una visión despersonalizada de los tiempos pretéritos que dificulta la comprensión de la complejidad de la historia.

Cabe tener en cuenta que las memorias que relataban familiares, vecinos y conocidos resultaron importantísimas para la interiorización de los aprendizajes que habían adquirido en la asignatura de Historia. No obstante, la mayor dificultad a la que nos tuvimos que enfrentar fue tratar de evitar que nuestros alumnos cayesen en el error de confundir memoria e historia, conceptos que en ocasiones se imbrican y cuya confusión ha generado una enorme cantidad de críticas y debates entre los especialistas en Historia y en Didáctica de la Historia (Prats, 2008).

En cualquier caso, estas fuentes históricas ayudan al estudiante de Historia a repensar por completo su concepción de la disciplina; además, contribuyen al fomento de la reflexión sobre esta materia, procesando la información y pensando de forma crítica. Gracias al uso de las fuentes atendidas como recurso educativo en la enseñanza reglada de la Historia, han tenido lugar sesiones de aprendizaje más participativas que han permitido lograr un conocimiento más significativo de la materia, así como el desarrollo de habilidades reflexivas y de investigación entre los alumnos. Igualmente, han dado lugar a un aprendizaje activo en que el alumno ha construido su propio conocimiento histórico a partir de estos recursos que sustituyen al método expositivo tradicional, que todavía es hegemónico en la enseñanza de la Historia en Educación Secundaria Obligatoria.

En síntesis, la Historia ha contribuido a la reflexión, confrontación de informaciones y desarrollo del pensamiento crítico (Parra, 2013). Igualmente, al escuchar a sus allegados han entendido el significado de esta disciplina, su utilidad y han visto que los actos que sus parientes o allegados hicieron en el pasado, tienen consecuencias en el presente. Han comprendido que la Historia no es algo alejado en el tiempo y ajeno a ellos, sino que está muy presente en su vida cotidiana dado que han asumido la cercanía de esta materia por lo familiares que les resultaban los entrevistados. Estas razones, y lo sugerente que puede ser para ellos el uso de una metodología nueva, han contribuido a interesar a nuestros alumnos por la Historia.

\section{Bibliografía}

AUBERT, A; FLECHA, A; GARCÍA, C; FLECHA, R. y RACIONERO, S. (2008). El aprendizaje dialógico en la sociedad de la Información. Barcelona: Hipatia Editorial.

BENADIBA, L. y PLOTINSKY,D. (2009). Historia oral. Construcción del archivo histórico escolar: una herramienta para la enseñanza de las ciencias sociales. Madrid: CEP.

CHARTIER, R. (1995). El mundo como representación; historia cultural, 
entre práctica y representación. Barcelona: Gedisa.

FRASER, R. (2001). Recuérdalo tú y recuérdalo a otros. Historia oral de la guerra civil española. Barcelona: Crítica.

GOLEMAN, D. (2006). Inteligencia emocional. Barcelona: Kairós.

JANER, G. y SANTOS, D. (1990). Fuentes orales y educación. Barcelona: Pirenne.

JARDÓN, X. X; LÓPEZ, M. J. y SOUTELO, R. (1998). “¡Eu tamén son vella e recordo!" La fuente oral en la recuperación del pasado: reflexiones metodológicas para una historia sociocultural, Espacio, Tiempo y Forma. Historia Contemporánea, 343-364.

JOHNSON, D. W. y JOHNSON, R. T. (1999). Aprender juntos y solos. Buenos Aires: Aique.

JOUTARD, PH. (1986). Esas voces que nos llegan del pasado. México: FCE.

MEC (2007). REAL DECRETO 1631/2006, de 29 de diciembre, por el que se establecen las enseñanzas mínimas correspondientes a la Educación Secundaria Obligatoria, Madrid, MEC-Secretaría General de Educación.

MIRALLES MONSERRAT, J. (1985). La història oral. Qüestionari i guia didáctica. Palma de Mallorca: Moll.

MONTEAGUDO, J. (2013). Propuesta para el tratamiento de las migraciones a través de fuentes orales en Educación Secundaria. Clío. History and History teaching, $\mathrm{n}^{\circ} 39$.
NAVARRO DOMÍNGUEZ, J. M. (2008). La práctica de la investigación histórica como vía para iniciar el trabajo en competencias en Ciencias Sociales. Íber. Didáctica de las Ciencias Sociales, Geografía e Historia, $\mathrm{n}^{\circ}$ 56, 53-62.

NÚÑ̃EZ, M. G. (1990). La Historia, las fuentes orales y la enseñanza: teoría y práctica. Espacio, Tiempo y Forma. Historia Contemporánea, $\mathrm{n}^{\circ}$ 3, 43-56.

PARRA MONSERRAT, D. (2013). La conceptualización de la Historia escolar y sus implicaciones didácticas. Un estudio a partir del recuerdo de estudiantes de BUP. Didáctica de las Ciencias experimentales y Sociales, $\mathrm{n}^{\circ}$ 27, 3-21. DOI: 10.7203/DCES.27.2653

PRATS, J. (2008). Memoria histórica "versus" historia enseñada. Íber. Didáctica de las Ciencias Sociales, Geografía e Historia, ${ }^{\circ}$ 55, 5-8.

PRATS, J. (2001). Enseñar Historia: Notas para una didáctica renovadora. Mérida, Junta de ExtremaduraConsejería de Educación, Ciencia y Tecnología-Dirección General de Ordenación, Renovación y Centros.

RAMOS LÓPEZ, S. y LORENZO SANTANA, J. R. (1996). ¡Cuanto saben los abuelos! Una investigación sobre el patrimonio oral. Cuadernos de Pedagogía, n 243, 29-31.

SALLÉS, N. (2010). La enseñanza de la historia a través del aprendizaje por descubrimiento: evolución del proyecto treinta años después. Enseñanza de las Ciencias Sociales, $\mathrm{n}^{\circ}$ 10, 3-10. 
SÁNCHEZ CARRERA, M. C. (1991). La utilización de las fuentes orales en la didáctica de las Ciencias Sociales. Investigación en la escuela, $\mathrm{n}^{\circ} 13,119$ 120.

SANTOS BURGALETA, M y SANTOS ESCRIBANO, F. (2011). Memoria, vida, aprendizaje: competencias básicas y fuentes orales en el aula de Ciencias Sociales. Revista de Claseshistoria, Publicación digital de Historia y Ciencias Sociales, 275.

SANTIESTEBAN, A. (2009). Cómo trabajar en clase la competencia social y ciudadana. Aula de Innovación Educativa, $\mathrm{n}^{\circ} 187,12-15$.

SEPÚLVEDA, G. (2000). Historia oral en el aula. Clío. History and History teaching, $\mathrm{n}^{\circ} 15$.
THOMPSON, P. (1988). Historia oral. La voz del pasado. Valencia: Edicions Alfons el Magnànim.

VALLS MONTÉS, R. (2007). La Guerra Civil española y la dictadura franquista: las dificultades del tratamiento escolar de un tema potencialmente conflictivo. Enseñanza de las ciencias sociales: revista de investigación, $\mathrm{n}^{\mathrm{0}}$ 6, 61-74.

VILANOVA, M. (1985). La història oral: una forma de dialogar. En D. LLOPART; J. PRAT y L. PRATS (eds.), La cultura popular a debat (128133). Barcelona: Fundació de Cultura Popular-Editorial Altafulla. 
